\title{
Expressive Footwear for Computer-Augmented Dance Performance
}

\author{
Joseph A. Paradiso and Eric Hu \\ MIT Media Laboratory \\ 20 Ames St. \\ Cambridge, MA 02139 \\ (+1)-617-253-8988 \\ joep,human@media.mit.edu
}

\begin{abstract}
A sensor system is described for instrumenting a pair of dancing shoes in order to capture many expressive degrees of freedom and use them to drive music synthesizers and computer graphics in a real-time performance. Dynamic pressure is measured at three points in the shoe sole, as are the bend of the sole, pitch and yaw shoe angles, and translational shoe positions. Data will be transmit across a 19.2 kbaud wireless link, enabling updates at $10 \mathrm{msec}$ intervals.
\end{abstract}

Keywords: Musical gesture sensing, body suit, shoe sensors, foot sensing, computer dance performance.

\section{Introduction}

Technologies under development for wearable computer systems can revolutionize several fields of artistic performance, such as theater or dance. Already, several artists have used body suits in computer-augmented performance [1,2]. Most of these devices merely employ embedded mechanical switches or piezoelectric sensors to detect simple strikes at several body locations. Others, such as in the Yamaha Miburi system [3], also use bend sensors to give continuous estimates of limb positions. None of these systems have attempted to instrument shoes with any degree of versatility, although the Miburi shoes provide piezoelectric triggers. Since the feet of a well-trained dancer are highly expressive appendages, an intimate computeraugmented performance needs realtime measurements of the many continuous parameters that can be acquired in the footwear. This paper describes a suite of sensors that we are now implementing into a set of dancing sneakers. As the signal conditioning needs are minimal and the processors and transmitters required are very small, we are building all electronics directly onto the shoe, avoiding difficulties with tethers running to a central communications unit.

Figure 1 shows a diagram of the shoe instrumented with a sensor array; we are now using a Capezio Dance Sneaker for the actual implementation. All sensors and subsystems are described below:

\section{Sensors and Electronics}

Two piezoelectric pads ("1" \& "2" in Fig. 1) made from flat, laminated sheets of PVDF (polyvinylidene fluoride) polymer [4] are placed at the front of the shoe sole, below the regions covered by the big and small toes. One PVDF pad ("3" in Fig. 1) is placed at the heel. PVDF has already been inserted into a set of shoes to measure footfalls for wearable computing applications [5]; the signals are several volts into a high-impedance ( $\geq 10 \mathrm{MegOhm})$ load, thus require only simple buffering before digitization. The two front pads will measure differential toe pressure, while the back pad will measure dynamic pressure at the heel. Although PVDF does not provide a steady-state force measurement, a dancer is usually in motion, and the most important performance features arise from dynamics.

We measure the sole deflection with a bidirectional FSR strip ("4" in Fig. 1) optimized for bending response [6], placed across the center of the sole where most bend occurs.

A dancer will often twist the foot in pitch $(\theta)$, independently of bending the sole (as measured above). The pitch coordinate is measured by a micromechanical accelerometer used as a tilt sensor ("6" in Fig. 1), where it senses the pitch component of the gravitational acceleration vector $(\mathrm{g})$. Both single and dual-axis devices are commonly available in small packages, with resolutions at the milli-g

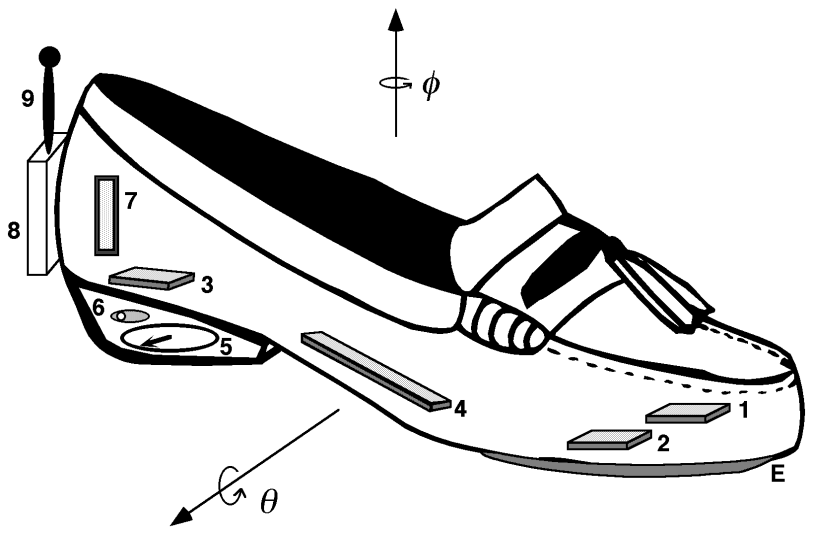

Figure 1. Shoe-borne instrumentation 


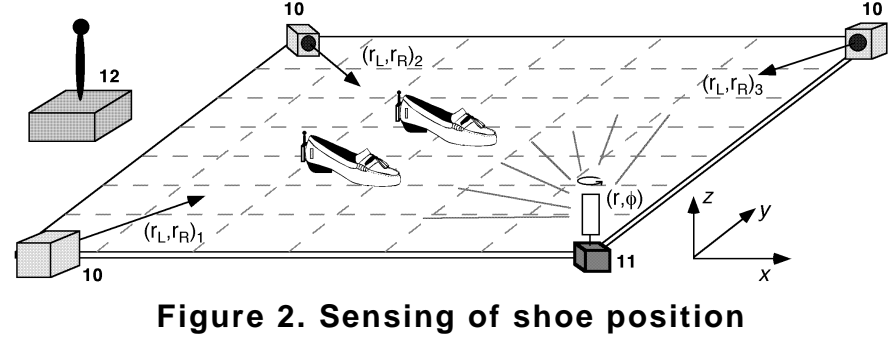

level [7]. Although the kinematics of the foot preclude the roll angle from being fully articulated, a dual-axis accelerometer (e.g., the ADXL202) will also measure this quantity. Besides responding to tilt, these accelerometers will also detect changes in foot velocity, plus give very clear indications and profiles of jumps and impacts.

The yaw coordinate $(\phi)$ will change as the dancer turns throughout the performance space. This can be measured directly (at least when the foot is oriented flat down) by embedding a small electronic compass into the sole ("5" in Fig. 1). We are now using a compact, magnetic vector sensor based on a permalloy bridge [8] to estimate bearing from the Earth's magnetic field. To obtain better response, we are also exploring the installation of a compact, micromechanical gyroscope [7]; any gyro drift can be compensated by the compass measurements.

Since signal conditioning requirements are minimal for this system, all electronics can be housed in a small compartment outside the shoe ("8" in Fig. 1). This will include a minimal embedded controller with 8-bit A/D converter to $\log$ and serialize the sensor data (e.g., a PIC 16C71 from Microchip Systems [9]), an analog multiplexer, battery adequate for at least 30 minutes of continuous operation, and a low-power RF transmitter, such as the TXM series of circa $400 \mathrm{MHz}$ FM devices from Abacom Technologies [10], which occupy a 1 x $3 \mathrm{~cm}$. surface-mount PC card. Since these transmitters can pass $20 \mathrm{~K}$ bits/second, the sensor data can be updated at roughly $10 \mathrm{msec}$ intervals. Because the range is so limited, a small antenna ("9" in Fig. 1) can either extend from the controller compartment, as in the figures, or be realized as a simple wire laminated onto the shoe itself. To enable continuous data transmission without the need of synchronization, both left and right feet will transmit on different RF carriers. These signals will be received and processed at a base station ("12" in Fig. 2), which analyzes the data and presents relevant features to the computers controlling the music and other aspects of the performance.

Translational position of the shoes can be measured by a scanning laser rangefinder (11 in Fig. 2) or directly via active sonar. We are mounting a strip of PVDF on the outside of the shoe ("7" in Fig. 1) to detect ultrasound pulses from sonar transmitters at the stage perimeter (" 10 " in Fig. 2). When transmitting its raster of acquired data, the microcontroller will add a set of bytes that specify the relative time at which the sonar ping were received. Each transmitter pings at a different frequency, thus all can fire simultaneously. Since PVDF is inherently broadband, its frequency response and selectivity can be set through a simple electronic filter.

Electric field sensing [11] will directly monitor the elevation of each foot; a copper strip above the sole transmits at $50-100 \mathrm{kHz}$, and the strength of the signal capacitively induced onto electrodes mounted atop the stage indicates the vertical shoe displacement.

\section{Conclusions}

A simple suite of compact, commercially-available sensors can easily be built into a shoe to measure many degrees of expression, together with a small microprocessor and wireless transmitter. This system will be used in a series of computer-augmented dance performances.

\section{Acknowledgments}

We are grateful to our colleagues at the MIT Media Laboratory for many discussions, especially Tod Machover, who has long been interested in expressive digital footwear. We appreciate the dance advice and prototype trials by Yuying Chen, and thank Jack Memishian from Analog Devices for donating the ADXL202's and Vic Chatigny of AMP Sensors for donating the PVDF. We acknowledge the support of the Things That Think Consortium and our other sponsors at the MIT Media Laboratory.

\section{References}

[1] L. Anderson, Stories from the Nerve Bible, HarperCollins, NY, 1994.

[2] M. Coniglio, Troika Ranch, The MidiDancer system, See: "http://www.art.net/ troika/mididancer.html".

[3] Yamaha Corporation, The Miburi performance system, See: "http://www.yamaha.co.jp/news/96041001.html".

[4] J. Paradiso, "The Interactive Balloon: Sensing, Actuation, and Behavior in a Common Object," IBM Systems Journal, 35(3\&4), 1996, pp. 473-487.

[5] S. Mann, "Smart Clothing: The Wearable Computer and WearCam," Personal Technologies, V. 1(1), 1997, pp. 21-27.

[6] FLX01 sensor from The Images Co., Staten Island, NY.

[7] C. Verplaetse, "Inertial Proprioceptive Devices: Selfmotion-sensing Toys and Tools," IBM Systems Journal, 35(3\&4), 1996, pp. 639-650.

[8] Honeywell, Inc., Solid State Electronics Center, Plymouth, MN, Magnetic Sensor Products.

[9] See "http://www.microchip.com/".

[10] Abacom Technologies, Etobicoke, Ontario, Canada.

[11] J. Paradiso, N. Gershenfeld, "Musical Applications of Electric Field Sensing," Computer Music Journ. 21(2), 1997. 